\title{
KRAVTSIV V. V.
}

\section{ANALOGUES OF THE NEWTON FORMULAS FOR THE BLOCK-SYMMETRIC POLYNOMIALS ON $\ell_{p}\left(\mathbb{C}^{s}\right)$}

\begin{abstract}
The classical Newton formulas gives recurrent relations between algebraic bases of symmetric polynomials. They are true, of course, for symmetric polynomials on infinite-dimensional Banach sequence spaces.

In this paper, we consider block-symmetric polynomials (or MacMahon symmetric polynomials) on Banach spaces $\ell_{p}\left(\mathbb{C}^{s}\right), 1 \leq p \leq \infty$. We prove an analogue of the Newton formula for the blocksymmetric polynomials for the case $p=1$. In the case $1<p$ we have no classical elementary block-symmetric polynomials. However, we extend the obtained Newton type formula for $\ell_{1}\left(\mathbb{C}^{s}\right)$ to the case of $\ell_{p}\left(\mathbb{C}^{s}\right), 1<p \leq \infty$, and in this way we found a natural definition of elementary block-symmetric polynomials on $\ell_{p}\left(\mathbb{C}^{s}\right)$.

Key words and phrases: symmetric polynomials, block-symmetric polynomials, algebraic basis, Newton's formula.
\end{abstract}

Vasyl Stefanyk Precarpathian National University, 57 Shevchenka str., 76018, Ivano-Frankivsk, Ukraine

E-mail: maksymivvika@gmail.com

\section{INTRODUCTION}

Let $X$ be a Banach space, and let $\mathcal{P}(X)$ be the algebra of all continuous polynomials defined on $X$. Let $\mathcal{P}_{0}(X)$ be a subalgebra of $\mathcal{P}(X)$. A sequence $\left(Q_{i}\right)_{i}$ of polynomials is called an algebraic basis of $\mathcal{P}_{0}(X)$ if for every $P \in \mathcal{P}_{0}(X)$ there is a unique polynomial $q \in \mathcal{P}\left(\mathbb{C}^{n}\right)$ for some $n$ such that $P(x)=q\left(Q_{1}(x), \ldots, Q_{n}(x)\right)$. In other words, if $Q$ is mapping $x \in$ $X \rightsquigarrow\left(Q_{1}(x), \ldots, Q_{n}(x)\right) \in \mathbb{C}^{n}$, then $P=q \circ Q$ and this representation is unique. Subalgebras of polynomials with countable algebraic bases were considered by many authors (see e. g. $[4,8,9,11,12])$. Typical examples of such kind of algebras are algebras of polynomials which are invariant with respect to a (semi)group $\mathcal{S}$ of operators on $X$. If $X$ has an unconditional basis $\left(e_{n}\right)$, we can consider the group $\mathcal{S}=S_{\infty}$ of all permutations of natural numbers $\mathbb{N}$ acting on $X$ by

$$
\sigma: x=\sum_{n=1}^{\infty} x_{n} e_{n} \rightsquigarrow \sum_{n=1}^{\infty} x_{\sigma(n)} e_{n} .
$$

$S_{\infty}$-invariant polynomials on $X$ are called symmetric. Symmetric polynomials and analytic functions on $\ell_{p}$ were investigated in $[1-3,5,6,8]$. Linear bases of symmetric polynomials on $\ell_{1}$ were considered in [7].

Let $\mathcal{P}_{S}\left(\ell_{p}\right)$ be the algebra of all symmetric polynomials on $\ell_{1}$. In [10], it is proved that polynomials

$$
F_{k}=\sum_{i=1}^{\infty} x_{i}^{k}
$$

$\mathrm{y} \triangle \mathrm{K} 517.98$

2010 Mathematics Subject Classification: 46J15, 46E10, 46E50. 
$k \geq\lceil p\rceil$ form an algebraic basis in $\mathcal{P}_{s}\left(\ell_{p}\right)$, where $\lceil p\rceil$ is the smallest integer, greater than $p$. Polynomials $F_{k}$ are called power symmetric polynomials. In the case $p=1$ we can consider another natural algebraic basis in $\mathcal{P}_{S}\left(\ell_{1}\right)$, which is called the basis of elementary symmetric polynomials, $\left\{G_{k}\right\}_{k=1}^{\infty}$,

$$
G_{k}=\sum_{i_{1}<i_{2}<\ldots<i_{k}}^{\infty} x_{i_{1}} x_{i_{2}} \ldots x_{i_{k}}
$$

The relation between power symmetric polynomials and elementary symmetric polynomials can be given by the well-known Newton formulas (see, e.g., [17]):

$$
n G_{n}=F_{1} G_{n-1}-F_{2} G_{n-2}+F_{3} G_{n-3}-\ldots+(-1)^{n-2} F_{n-1} G_{1}+(-1)^{n-1} F_{n}, \quad n \in \mathbb{N} .
$$

In the case $p>1$ we have no elementary symmetric polynomials, because the series (1) does not converge for any $k$. But putting in the Newton formulas $F_{k}=0$ for $k<p$, we can define elementary symmetric polynomials on $\ell_{p}$ by

$$
G_{n}^{(p)}=\sum_{k=\lceil p\rceil}^{n-\lceil p\rceil}(-1)^{k-1} F_{k} G_{n-k} .
$$

It is easy to check that the sequence $\left\{G_{n}^{(p)}\right\}_{n>p}$ forms an algebraic basis in $\mathcal{P}_{S}\left(\ell_{p}\right)$.

There are other natural representations of $S_{\infty}$ in Banach spaces with bases. For example, if $\mathcal{X}$ is a directs sum of infinite many of "blocks" which are copies of a Banach space $X$, then $S_{\infty}$ acts permutating the "blocks". For this case we can consider the algebra of block-symmetric analytic functions consisting of invariants of this group. Note that this algebra is much more complicated and in the finitely-dimensional case has no algebraic basis (see, e.g., $[15,19]$ ).

A generalization of the Newton formula for block-symmetric polynomials on $\ell_{1}\left(\mathbb{C}^{2}\right)$ was proved in [13]. In this paper we propose a generalization of this formula for block-symmetric polynomials on $\ell_{p}\left(\mathbb{C}^{s}\right)$.

\section{Main Result}

Let us denote by $\ell_{p}\left(\mathbb{C}^{s}\right), 1 \leq p<\infty$, the vector space of all sequences

$$
x=\left(x_{1}, x_{2}, \ldots, x_{m}, \ldots\right),
$$

where $x_{j}=\left(x_{j}^{(1)}, \ldots, x_{j}^{(s)}\right) \in \mathbb{C}^{s}$ for $j \in \mathbb{N}$, such that the series $\sum_{j=1}^{\infty} \sum_{r=1}^{s}\left|x_{j}^{(r)}\right|^{p}$ is convergent. The space $\ell_{p}\left(\mathbb{C}^{s}\right)$ with norm

$$
\|x\|=\left(\sum_{j=1}^{\infty} \sum_{r=1}^{s}\left|x_{j}^{(r)}\right|^{p}\right)^{1 / p}
$$

is a Banach space. A polynomial $P$ on the space $\ell_{p}\left(\mathbb{C}^{s}\right)$ is called block-symmetric (or vectorsymmetric) if

$$
P\left(x_{1}, x_{2}, \ldots, x_{m}, \ldots\right)=P\left(x_{\sigma(1)}, x_{\sigma(2)}, \ldots, x_{\sigma(m)}, \ldots\right)
$$

for every permutation $\sigma \in S_{\infty}$, where $x_{j} \in \mathbb{C}^{s}$ for all $j \in \mathbb{N}$. Let us denote by $\mathcal{P}_{\text {vs }}\left(\ell_{p}\left(\mathbb{C}^{s}\right)\right)$ the algebra of all block-symmetric polynomials on $\ell_{p}\left(\mathbb{C}^{s}\right)$. 
The algebra $\mathcal{P}_{v s}\left(\ell_{p}\left(\mathbb{C}^{s}\right)\right)$ was considered in $[14,16]$. Note that in Combinatorics, blocksymmetric polynomials on finite-dimension spaces are called MacMahon symmetric polynomials (see [18]).

For a multi-index $\mathbf{k}=\left(k_{1}, k_{2}, \ldots, k_{s}\right) \in \mathbb{Z}_{+}^{s}$ let $m=|\mathbf{k}|=k_{1}+k_{2}+\ldots+k_{s}$.

In [14] it was proved that polynomials

$$
H_{m}^{\mathbf{k}}(x)=H_{m}^{k_{1}, k_{2}, \ldots, k_{s}}(x)=\sum_{j=1}^{\infty} \prod_{\substack{r=1 \\|\mathbf{k}| \geq\lceil p\rceil}}^{s}\left(x_{j}^{(r)}\right)^{k_{r}}
$$

form an algebraic basis in $\mathcal{P}_{v s}\left(\ell_{p}\left(\mathbb{C}^{s}\right)\right), 1 \leq p<\infty$, where $x=\left(x_{1}, \ldots, x_{m}, \ldots\right) \in \ell_{p}\left(\mathbb{C}^{s}\right)$, $x_{j}=\left(x_{j}^{(1)}, \ldots, x_{j}^{(s)}\right) \in \mathbb{C}^{s}$.

In the case of the space $\ell_{1}\left(\mathbb{C}^{s}\right)$ there are elementary block-symmetric polynomials

$$
R_{m}^{\mathbf{k}}(x)=R_{m}^{k_{1}, k_{2}, \ldots, k_{s}}(x)=\sum_{\substack{i_{1}<\ldots<i_{k_{1}} \\ j_{1}<\ldots<j_{k_{2}} \\ l_{1}<\ldots<l_{k_{s}} \\ i_{k_{p}} \neq j_{k_{q}} \neq \ldots \neq l_{k_{r}}}}^{\infty} x_{i_{1}}^{(1)} \ldots x_{i_{k_{1}}}^{(1)} x_{j_{1}}^{(2)} \ldots x_{j_{k_{2}}}^{(2)} \ldots x_{l_{1}}^{(s)} \ldots x_{l_{k_{s}}}^{(s)},
$$

where $\left(x_{i}^{(1)}, x_{i}^{(2)}, \ldots, x_{i}^{(s)}\right) \in \mathbb{C}^{s}$.

Combining (2) and (3), we can get an analog of Newton's formula for block-symmetric polynomials on $\ell_{1}\left(\mathbb{C}^{s}\right)$.

Theorem 1. The following formula is true for the algebraic bases of symmetric polynomials on $\ell_{1}\left(\mathbb{C}^{s}\right)$.

$$
\begin{aligned}
n R_{n}^{k_{1}, k_{2}, \ldots, k_{s}} & =\sum_{\substack{|\mathbf{q}|=1 \\
k_{r} \geq q_{r}}} H_{1}^{q_{1}, q_{2}, \ldots, q_{s}} R_{n-1}^{k_{1}-q_{1}, k_{2}-q_{2}, \ldots, k_{s}-q_{s}} \\
& -\sum_{\substack{|\mathbf{q}|=2 \\
k_{r} \geq q_{r}}} \frac{2 !}{q_{1} ! q_{2} ! \ldots q_{s} !} H_{2}^{q_{1}, q_{2}, \ldots, q_{s}} R_{n-2}^{k_{1}-q_{1}, k_{2}-q_{2}, \ldots, k_{s}-q_{s}}+\ldots \\
& +(-1)^{n-2} \sum_{\substack{|\mathbf{q}|=n-1 \\
k_{r} \geq q_{r}}} \frac{(n-1) !}{q_{1} ! q_{2} ! \ldots q_{s} !} H_{n-1}^{q_{1}, q_{2}, \ldots, q_{s}} R_{1}^{k_{1}-q_{1}, k_{2}-q_{2}, \ldots, k_{s}-q_{s}} \\
& +(-1)^{n-1} \frac{n !}{k_{1} ! k_{2} ! \ldots k_{s} !} H_{n}^{k_{1}, k_{2}, \ldots, k_{s}},
\end{aligned}
$$

where $\mathbf{q}=\left(q_{1}, q_{2}, \ldots, q_{s}\right), R_{0}^{k_{1}, k_{2}, \ldots, k_{s}} \equiv 1$ and if $k_{r}<q_{r}$ for some $r=1, \ldots, s$, then $R_{m}^{k_{1}-q_{1}, k_{2}-q_{2}, \ldots, k_{s}-q_{s}} \equiv 0$.

Proof. Let us consider the polynomial $P\left(t_{1} x^{(1)}+t_{2} x^{(2)}+\ldots+t_{s} x^{(s)}\right)$, which is symmetric on the space $\ell_{1}$ with respect to simultaneously permutations of $t_{1} x_{i}^{(1)}+t_{2} x_{i}^{(2)}+\ldots+t_{s} x_{i}^{(s)}, i \geq 1$. Let us denote by $\widetilde{t x}=t_{1} x^{(1)}+t_{2} x^{(2)}+\ldots+t_{s} x^{(s)}$. For the algebraic bases $F_{k}(\widetilde{t x})$ and $G_{k}(\widetilde{t x})$ of this polynomial the Newton formula holds

$$
\begin{aligned}
n G_{n}(\widetilde{t x}) & =F_{1}(\widetilde{t x}) G_{n-1}(\widetilde{t x})-F_{2}(\widetilde{t x}) G_{n-2}(\widetilde{t x}) \\
& +F_{3}(\widetilde{t x}) G_{n-3}(\widetilde{t x})-\ldots+(-1)^{n-2} F_{n-1}(\widetilde{t x}) G_{1}(\widetilde{t x})+(-1)^{n-1} F_{n}(\widetilde{t x}) .
\end{aligned}
$$


Each of polynomials $F_{m}(\widetilde{t x})$ and $G_{m}(\widetilde{t x})$ can be represented as a linear combination of polynomials $H_{m}^{k_{1}, k_{2}, \ldots, k_{s}}(x)$ and $R_{m}^{k_{1}, k_{2}, \ldots, k_{s}}(x)$ respectively. Indeed,

$$
\begin{aligned}
G_{n}(\tilde{t x}) & =G_{n}\left(t_{1} x^{(1)}+t_{2} x^{(2)}+\ldots+t_{s} x^{(s)}\right) \\
& =\sum_{i_{1}<\ldots<i_{n}}\left(t_{1} x^{(1)}+t_{2} x^{(2)}+\ldots+t_{s} x^{(s)}\right)_{i_{1}} \ldots\left(t_{1} x^{(1)}+t_{2} x^{(2)}+\ldots+t_{s} x^{(s)}\right)_{i_{n}} \\
& =\sum_{p_{1}+p_{2}+\ldots+p_{s}=n} t_{1}^{p_{1}} t_{2}^{p_{2}} \ldots t_{s}^{p_{s}} R_{n}^{p_{1}, p_{2}, \ldots, p_{s}}(x)
\end{aligned}
$$

and

$$
\begin{aligned}
F_{n}(\widetilde{t x}) & =F_{n}\left(t_{1} x^{(1)}+t_{2} x^{(2)}+\ldots+t_{s} x^{(s)}\right)=\sum_{i=1}^{\infty}\left(t_{1} x^{(1)}+t_{2} x^{(2)}+\ldots+t_{s} x^{(s)}\right)_{i}^{n} \\
& =\sum_{k_{1}+k_{2}+\ldots+k_{s}=n} \frac{n !}{k_{1} ! k_{2} ! \ldots k_{s} !} t_{1}^{k_{1}} t_{2}^{k_{2}} \ldots t_{s}^{k_{s}} H_{n}^{k_{1}, k_{2}, \ldots, k_{s}}(x) .
\end{aligned}
$$

So each term of equality (5) can be represented by polynomials $H_{m}^{k_{1}, k_{2}, \ldots, k_{s}}$ and $R_{m}^{p_{1}, p_{2}, \ldots, p_{s}}$. Then we obtain

$$
\begin{aligned}
& F_{1}(\widetilde{t x}) G_{n-1}(\widetilde{t x})=\left(\sum_{k_{1}+k_{2}+\ldots+k_{s}=1} \frac{1 !}{k_{1} ! k_{2} ! \ldots k_{s} !} t_{1}^{k_{1}} t_{2}^{k_{2}} \ldots t_{s}^{k_{s}} H_{1}^{k_{1}, k_{2}, \ldots, k_{s}}(x)\right) \\
& \times\left(\sum_{p_{1}+p_{2}+\ldots+p_{s}=n-1} t_{1}^{p_{1}} t_{2}^{p_{2}} \ldots t_{s}^{p_{s}} R_{n-1}^{p_{1}, p_{2}, \ldots, p_{s}}(x)\right) \\
& =\sum_{\substack{k_{1}+k_{2}+\ldots+k_{s}=1 \\
p_{1}+p_{2}+\ldots+p_{s}=n-1}} \frac{1 !}{k_{1} ! k_{2} ! \ldots k_{s} !} t_{1}^{k_{1}+p_{1}} t_{2}^{k_{2}+p_{2}} \ldots t_{s}^{k_{s}+p_{s}} H_{1}^{k_{1}, k_{2}, \ldots, k_{s}}(x) R_{n-1}^{p_{1}, p_{2}, \ldots, p_{s}}(x), \\
& F_{r}(\widetilde{t x}) G_{n-r}(\widetilde{t x})=\left(\sum_{k_{1}+k_{2}+\ldots+k_{s}=r} \frac{r !}{k_{1} ! k_{2} ! \ldots k_{s} !} t_{1}^{k_{1}} t_{2}^{k_{2}} \ldots t_{s}^{k_{s}} H_{r}^{k_{1}, k_{2}, \ldots, k_{s}}(x)\right) \\
& \times\left(\sum_{p_{1}+p_{2}+\ldots+p_{s}=n-r} t_{1}^{p_{1}} t_{2}^{p_{2}} \ldots t_{s}^{p_{s}} R_{n-r}^{p_{1}, p_{2}, \ldots, p_{s}}(x)\right) \\
& =\sum_{\substack{k_{1}+k_{2}+\ldots+k_{s}=r \\
p_{1}+p_{2}+\ldots+p_{s}=n-r}} \frac{r !}{k_{1} ! k_{2} ! \ldots k_{s} !} t_{1}^{k_{1}+p_{1}} t_{2}^{k_{2}+p_{2}} \ldots t_{s}^{k_{s}+p_{s}} H_{r}^{k_{1}, k_{2}, \ldots, k_{s}}(x) R_{n-r}^{p_{1}, p_{2}, \ldots, p_{s}}(x) .
\end{aligned}
$$

If we substitute this equalities and equalities (6), (7) into (5) and equate multipliers at the all powers of $t_{i}, i=1, \ldots, s$ we obtain the required formula.

Note that equation (4) is invertible and so we have

$$
\begin{aligned}
\frac{n !}{k_{1} ! \ldots k_{s} !} H_{n}^{k_{1}, \ldots, k_{s}} & =\sum_{\substack{|\mathbf{q}|=n-1 \\
k_{r} \geq q_{r}}} \frac{(n-1) !}{q_{1} ! \ldots q_{s} !} H_{n-1}^{q_{1}, \ldots, q_{s}} R_{1}^{k_{1}-q_{1}, \ldots, k_{s}-q_{s}}+\ldots \\
& +(-1)^{n-1} \sum_{\substack{|\mathbf{q}|=2 \\
k_{r} \geq q_{r}}} \frac{2 !}{q_{1} ! \ldots q_{s} !} H_{2}^{q_{1}, \ldots, q_{s}} R_{n-2}^{k_{1}-q_{1}, \ldots, k_{s}-q_{s}} \\
& +(-1)^{n} \sum_{\substack{|\mathbf{q}|=1 \\
k_{r} \geq q_{r}}} H_{1}^{q_{1}, \ldots, q_{s}} R_{n-1}^{k_{1}-q_{1}, \ldots, k_{s}-q_{s}}+(-1)^{n+1} n R_{n}^{k_{1}, \ldots, k_{s}} .
\end{aligned}
$$


Let us rewrite formula (4) using multi-index notations. We denote by $\mathbf{k} !=k_{1} ! k_{2} ! \ldots k_{s}$ ! and by $\mathbf{k}-\mathbf{q}=\left(k_{1}-q_{1}, k_{2}-q_{2}, \ldots k_{s}-q_{s}\right)$. Also, we say that $\mathbf{k} \geq \mathbf{q}$ if and only if $k_{1} \geq q_{1}$, $k_{2} \geq q_{2}, \ldots, k_{s} \geq q_{S}$. Then (4) can be expressed by

$$
\begin{aligned}
n R_{n}^{\mathbf{k}} & =\sum_{\substack{|\mathbf{q}|=1 \\
\mathbf{k} \geq \mathbf{q}}} H_{1}^{\mathbf{q}} R_{n-1}^{\mathbf{k}-\mathbf{q}}-\sum_{\substack{|\mathbf{q}|=2 \\
\mathbf{k} \geq \mathbf{q}}} \frac{|\mathbf{q}| !}{\mathbf{q} !} H_{2}^{\mathbf{q}} R_{n-2}^{\mathbf{k}-\mathbf{q}}+\cdots+(-1)^{n-2} \sum_{\substack{|\mathbf{q}|=n-1 \\
\mathbf{k} \geq \mathbf{q}}} \frac{|\mathbf{q}| !}{\mathbf{q} !} H_{n-1}^{\mathbf{q}} R_{1}^{\mathbf{k}-\mathbf{q}} \\
& +(-1)^{n-1} \frac{n !}{\mathbf{k} !} H_{n}^{\mathbf{k}}=\sum_{j=1}^{n}(-1)^{j-1} \sum_{\substack{|\mathbf{q}|=j \\
\mathbf{k} \geq \mathbf{q}}} \frac{|\mathbf{q}| !}{\mathbf{q} !} H_{j}^{\mathbf{q}} R_{n-j}^{\mathbf{k}-\mathbf{q}}, \quad \text { where } n=|\mathbf{k}| .
\end{aligned}
$$

Comparing formula (8) with the classical Newton formula we can see that their are coincide if $s=1$.

Let us turn out to the space $\ell_{p}\left(\mathbb{C}^{s}\right)$. Taking into account formula (2) we can see that by definition, $H_{m}^{\mathbf{k}}=0$ in $\mathcal{P}_{v s}\left(\ell_{p}\left(\mathbb{C}^{s}\right)\right)$ if $|\mathbf{k}|<\lceil p\rceil$. So, using (8), we can define elementary blocksymmetric polynomials on $\ell_{p}\left(\mathbb{C}^{s}\right)$ by

$$
n R_{n}^{\mathbf{k}}=\sum_{j=\lceil p\rceil}^{n-\lceil p\rceil}(-1)^{j-1} \sum_{\substack{|\mathbf{q}|=j \\ \mathbf{k} \geq \mathbf{q}}} \frac{|\mathbf{q}| !}{\mathbf{q} !} H_{j}^{\mathbf{q}} R_{n-j}^{\mathbf{k}-\mathbf{q}}, \quad \text { where } \quad n=|\mathbf{k}| \geq\lceil p\rceil .
$$

Theorem 2. Elementary block-symmetric polynomials on $\ell_{p}\left(\mathbb{C}^{s}\right)$ defined by (9) form an algebraic basis of $n$-homogeneous polynomials $n \geq\lceil p\rceil$ in $\mathcal{P}_{v s}\left(\ell_{p}\left(\mathbb{C}^{s}\right)\right)$.

Proof. It is easy to see that equation (9) is invertible. So we have a bijection between polynomials $H_{n}^{\mathbf{q}}$ and $R_{n}^{\mathbf{q}}$. Since $\left\{H_{n}^{\mathbf{q}}\right\}_{n \geq\lceil p\rceil}$ is an algebraic basis in $\mathcal{P}_{v s}\left(\ell_{p}\left(\mathbb{C}^{s}\right)\right)$, so the set $\left\{R_{n}^{\mathbf{q}}\right\}_{n \geq\lceil p\rceil}$ is an algebraic basis in $\mathcal{P}_{v s}\left(\ell_{p}\left(\mathbb{C}^{s}\right)\right)$ too.

\section{REFERENCES}

[1] Alencar R., Aron R., Galindo P., Zagorodnyuk A. Algebra of symmetric holomorphic functions on $\ell_{p}$. Bull. Lond. Math. Soc. 2003, 35, 55-64. doi:10.1112/S0024609302001431

[2] Aron R., Falcó J., Maestre M. Separation theorems for group invariant polynomials. J. Geom. Anal. 2018, 28 (1), 393-404. doi:10.1007/s12220-017-9825-0.

[3] Aron R., Galindo P., Pinasco D., Zalduendo I. Group-symmetric holomorphic functions on a Banach space. Bull. Lond. Math. Soc. 2016, 48 (5), 779--796. doi:10.1112/blms/bdw043

[4] Chernega I., Holubchak O., Novosad Z., Zagorodnyuk A. Continuity and hypercyclicity of composition operators on algebras of symmetric analytic functions on Banach spaces. European Journal of Mathematics 2020, 6, 153--163. doi:10.1007/s40879-019-00390-z

[5] Chernega I., Galindo P., Zagorodnyuk A. Some algebra of symmetric analytic functions and their spectra. Proc. Edinburgh Math. Soc. 2012, 55, 125-142. doi:10.1017/S0013091509001655

[6] Chernega I., Galindo P., Zagorodnyuk A. The convolution operation on the spectra of algebras of symmetric analytic functions. J. Math. Anal. Appl. 2012, 395 (2), 569-577. doi:10.1016/j.jmaa.2012.04.087

[7] Chernega I., Zagorodnyuk A. Note on bases in algebras of analytic functions on Banach spaces. Carpathian Math. Publ. 2019, 11 (1), 42-47. doi:10.15330/cmp.11.1.42-47

[8] Galindo P., Vasylyshyn T., Zagorodnyuk A. Symmetric and finitely symmetric polynomials on the spaces $\ell_{\infty}$ and $L_{\infty}[0,+\infty]$. Math. Nachr. 2018, 291 (11-12), 1712-1726. doi:10.1002/mana.201700314

[9] Galindo P., Vasylyshyn T., Zagorodnyuk A. Analytic structure on the spectrum of the algebra of symmetric analytic functions on $L_{\infty}$. RACSAM 2020, 114 (56), 13 pages. doi:10.1007/s13398-020-00791-w

[10] Gonzalez M., Gonzalo R., Jaramillo J. Symmetric polynomials on rearrangement invariant function spaces. J. Lond. Math. Soc. 1999, 59 (2), 681-697. doi:10.1112/S0024610799007164 
[11] Halushchak S. Spectra of some algebras of entire functions of bounded type, generated by a sequence of polynomials. Carpathian Math. Publ. 2019, 11 (2), 311--320. doi:10.15330/cmp.11.2.311-320

[12] Jawad F., Zagorodnyuk F. Supersymmetric Polynomials on the Space of Absolutely Convergent Series. Symmetry 2019, 11 (9), 1111. doi:10.3390/sym11091111

[13] Kravtsiv V. The Analogue of Newton's Formula for Block-Symmetric Polynomials. International Journal of Mathematical Analysis 2016, 10 (7), 323-327. doi:10.12988/ijma.2016.617

[14] Kravtsiv V., Vasylyshyn T., Zagorodnyuk A. On Algebraic Basis of the Algebra of Symmetric Polynomials on $l_{p}\left(C^{n}\right)$. J. Funct. Spaces 2017, 2017, Article ID 4947925, 8 pages. doi:10.1155/2017/4947925

[15] Kravtsiv V.V., Zagorodnyuk A.V. Multiplicative Convolution on the Algebra of Block-Semmetric Analytic Functions. J. Math. Sci. (N.Y.) 2020, 246 (2), 245-255. doi:10.1007/s10958-020-04734-Z

[16] Kravtsiv V.V., Zagorodnyuk A.V. On algebraic bases of algebras of block-symmetric polynomials on Banach spaces. Mat. Stud. 2012, 37 (1), 109-112.

[17] Macdonald I.G. Symmetric Functions and Orthogonal Polynomials. University Lecture Series, Vol. 12, AMS, Providence, RI, 1997.

[18] Rosas M.H. MacMahon symmetric functions, the partition lattice, and Young subgroups. J. Combin. Theory Ser. A 2001, 96, 326-340. doi:10.1006/jcta.2001.3186

[19] Zagorodnyuk A., Kravtsiv V. Symmetric polynomials on the product of Banach spaces. Carpathian Math. Publ. 2010, 2 (1), 59-71. (in Ukrainian)

Received 04.03.2020

Кравців В.В. Аналог формули Ньютона для блочно-симетричних поліномів на $\ell_{p}\left(\mathbb{C}^{n}\right) / /$ Карпатські матем. публ. - 2020. - Т.12, №1. - С. 17-22.

Класичні формули Ньютона задає рекурентні співвідношення між алгебраїчними базисами симетричних поліномів. Ці формули залишаються правильними і для симетричних поліномів на нескінченновимірних банахових просторах послідовностей.

В цій статті ми розглялаємо блочно-симетричні поліноми (або симетричні полінома Макмахона) на банахових просторах $\ell_{p}\left(\mathbb{C}^{s}\right), 1 \leq p \leq \infty$. Ми доводимо аналог формули Ньютона Аля блочно-симетричних поліномів у випадку $p=1$. У випадку $1<p$ немає класичних елементарних блочно-симетричних поліномів. Проте ми продовжили отриману формулу типу Ньютона для $\ell_{1}\left(\mathbb{C}^{s}\right)$ на випадок $\ell_{p}\left(\mathbb{C}^{s}\right), 1<p \leq \infty, \mathrm{i}$ в такий спосіб, запропонували природнє означення елементарних блочно-симетричних поліномів на $\ell_{p}\left(\mathbb{C}^{s}\right)$.

Ключові слова і фрази: симетричні поліноми, блочно-симетричні поліноми, алгебраїчний базис, формула Ньютона. 